\title{
The Periodic Operation of a Semiconductor Gas Sensor and Its Application to the Discrimination of Inflammable Gases ${ }^{\dagger}$
}

\author{
Byeongdeok YeA*, Tomoyuki OsaKi*, \\ Kazunori SugaharA* and Ryosuke KONISHI*
}

\begin{abstract}
A new operation method of a semiconductor gas sensor is proposed to improve the gas selectivity and temperaturehumidity characteristics of the sensor. In the method the heater voltage of the sensor is changed periodically, named periodic operation, for a certain time interval after inflammable gases were introduced. The method is examined in discriminating five kinds of inflammable gases with a three-layered BP neural network, and high discrimination rate (average $98 \%$ ) is achieved.
\end{abstract}

Key Words: semiconductor gas sensor, periodic operation, transient response, neural network

\section{Introduction}

Semiconductor gas sensors based on tin dioxide $\left(\mathrm{SnO}_{2}\right)$ are widely used in many applications nowadays because of their advantages such as small size, light weight, high sensitivity and long durability. However, the gas selectivity of the sensors still remained an unsolved problem in spite of numerous research that have been invested in improving it ${ }^{1) \sim 3 \text { ) }}$.

Recently, we have reported several researches on gas and/or odor sensing systems which show good performances in discriminating gas and/or odor samples having different characteristics ${ }^{4), 5)}$. However, for the gas samples of similar characteristics, the discrimination rates were considerably lower than those of different characteristics, and the temperature-humidity dependence of the sensors is regarded as the major reason of such results.

In this paper, we propose periodic operation of semiconductor gas sensors to improve the gas selectivity of the sensor. The proposed method is examined in discriminating five kinds of inflammable gases, and the effectiveness of the method is discussed based on the results.

\section{Periodic Operation of a Semiconduc- tor Gas Sensor}

There has been two kinds of operating methods up to date for semiconductor gas sensors commercially available; one is utilizing steady-state responses of the sensors, and the other the transient responses.

$\uparrow$ Presented at the 42nd Spring Meeting of the Japan Society of Applied Physics and Related Societies (1995. 3)

* Faculty of Engineering, Tottori University, 4-101, Minami, Koyama-Cho, Tottori

(Received October 23, 1996)

(Revised October 24, 1997)
When conventional uses are concerned, it is sufficient to operate the sensors at the regular heater voltages as shown in Fig. 1(a). This operating method is relatively simple one because it needs no additional processors or circuits to control the heater voltage. However, the responses of the sensor are changed even in the same atmosphere due to the ambient temperature-humidity variations. The method is not applicable for gas discrimination as long as one sensor is involved, consequently.

The second one is utilizing the transient response patterns of the sensors as illustrated in Fig. 1(b). The heater voltage of the sensor is changed once after the responses are settled in steady state to obtain more information than the former operating method. Even though the selectivity of the sensor is improved considerably with the method, there still remain difficulties in discriminating gas samples having similar characteristics ${ }^{4) \sim 6)}$

To cope with such difficulties, we propose periodic operation of the sensors in this paper. The periodic operation means the periodic change of heater voltage of the sensor for a certain time interval after inflammable gases were introduced as shown in Fig. 1(c). In the proposed method the transient responses are utilized more actively, that is, not only the downwards transient responses but also the upwards transient responses are utilized, and it can be expected that more information can be obtained from the responses, consequently.

The efficiency of the proposed method is examined in discriminating inflammable gases of different characteristics as well as of similar characteristics.

\section{Experimental}

The same experimental apparatus as in our previous report ${ }^{5)}$ was utilized.

Hydrogen, town gas, butane, liquid petroleum gas (LP 


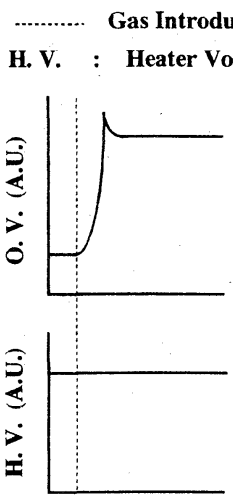

(a)
-.-. Heater Voltage Change

O. V. : Output Voltage

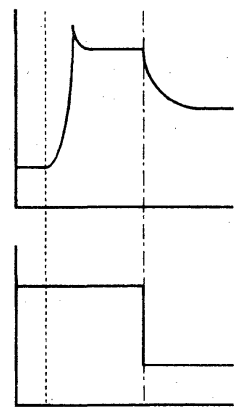

(b)

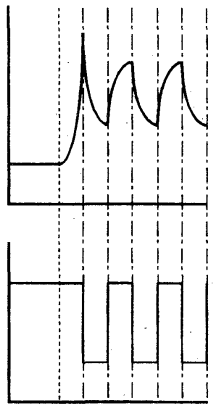

(c)
Fig. 1 Schematic diagram of operation methods for semiconductor gas sensors.

gas, hereinafter) and methane were introduced as gas samples, and the response patterns of the sensor were measured as voltage variation on a series resistance connected to it. The samples were selected elaborately to examine the performance of the proposed method in discriminating gases of similar characteristics; hydrogen and town gas compose a pair of similar gas sample because the latter in Tottori, Japan contains a large quantity of the former (more than 40\%), and butane and LP gas form another pair because of similar reason.

The concentration of the introduced gas was maintained at $1000 \mathrm{ppm}$ during the measurement.

\section{Results and Discussion}

Figure 2 illustrates the typical heater voltage and corresponding response patterns of TGS 824 operated periodically. Gas samples were introduced at $3 \mathrm{~s}$ and the heater voltage started to vary at $8 \mathrm{~s}$; the period of voltage variation was $16 \mathrm{~s}$, the duration of the variation was 48 $\mathrm{s}$, that is, three periods, and the high-level and low-level heater voltages are $5 \mathrm{~V}$ and $3 \mathrm{~V}$, respectively. The levels of the heater voltage are determined by considering the surface temperatures of the sensor which show maximum sensitivity for the selected gases. In other words, the surface temperatures of the sensor are around $450^{\circ} \mathrm{C}$ at $5 \mathrm{~V}$ and around $200^{\circ} \mathrm{C}$ at $3 \mathrm{~V}$, which correspond to the temperatures showing maximum sensitivity for methane and hydrogen, respectively ${ }^{7)}$.

Response patterns of the first period ( 0 to $16 \mathrm{~s}$ ) showed unstable characteristics because of the great changes due to the gas introduction; on the other hand, the patterns after the 1st period were stable and reproducible, and seemed to be usable for discrimination. Furthermore, because there appeared periodicity on the patterns, only a period of data would be sufficient for discrimination. We

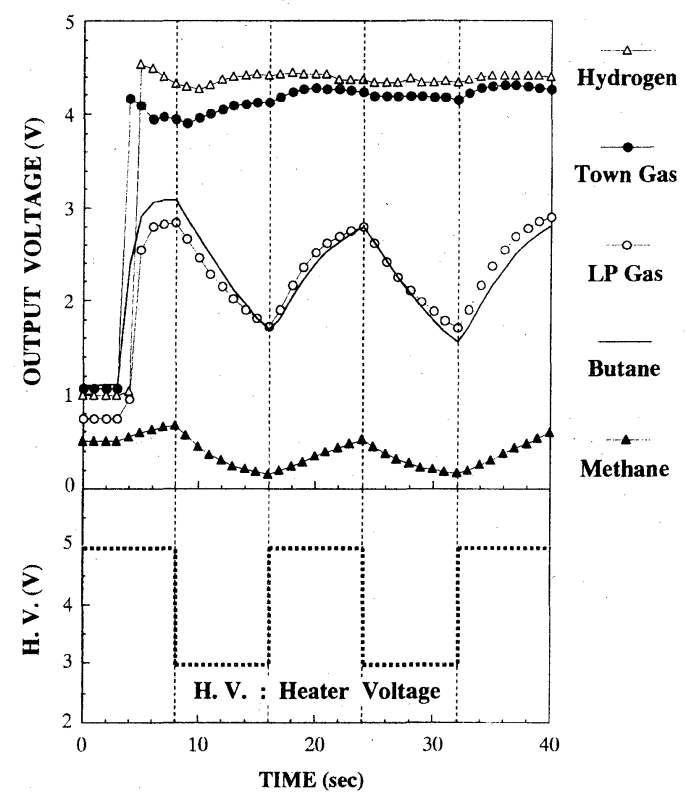

Fig. 2 Typical example of periodic heater voltage and corresponding response patterns of TGS824 for introduced gas samples.

utilized the 16 points of data of the second period for discriminating the introduced gases.

In general, there would be aging effect on the sensor surface by the abrupt and long-term change of the surface temperature, which could result the drift or variance of sensor characteristics. However, in this case, the heater voltage is changed only for 3 periods ( $48 \mathrm{~s}$ ) when the gas is introduced; otherwise, it is kept on $5 \mathrm{~V}$. Therefore, we expect that such effect would not affect the sensor characteristics.

Even though the patterns of the similar gas pair resembled each other, small differences appeared on the patterns, and it is expected that the discrimination of them can be achieved by emphasizing those differences.

We measured the response patterns 150 times over 6 weeks for total gas samples, that is, prepared 30 patterns for each gas sample. We did not take any special care for the ambient temperature-humidity variation during the measurements because we wanted to examine the efficiency of the proposed method for such variation. The variations of temperature and relative humidity during the measurements were from 17 to $23^{\circ} \mathrm{C}$ and from 60 to $90 \%$, respectively. The response patterns showed some range of errors due to the variations; the maximum range of error was about $0.6 \mathrm{~V}$ in case of butane and LP gas, about $0.3 \mathrm{~V}$ in case of hydrogen and town gas, and about $0.4 \mathrm{~V}$ for methane.

It seems that the discrimination of the gas samples having similar characteristics is difficult because the response 


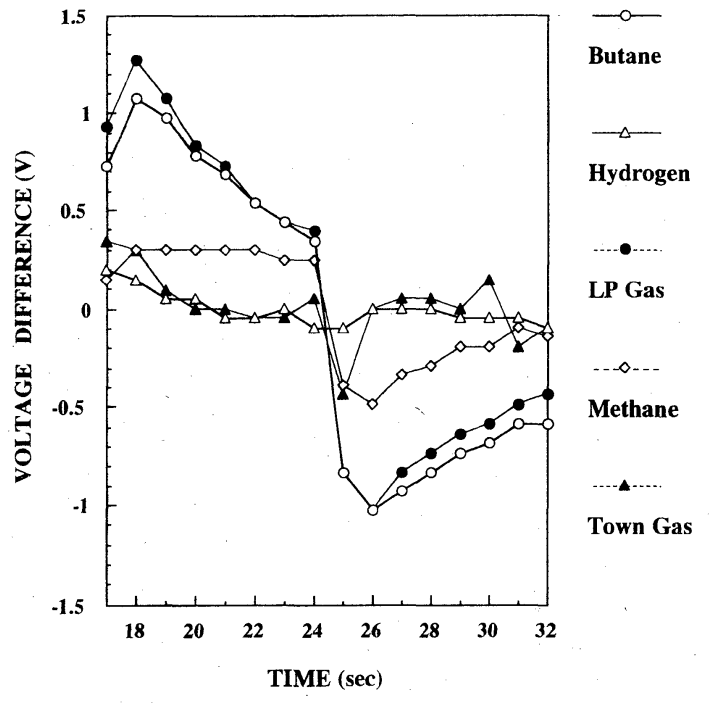

Fig. 3 Examples of input patterns for the neural network.

patterns of those samples will fold each other owing to the range of errors. However, the shape of the response patterns shows little change in spite of the range of errors, and, consequently, the discrimination of such similar samples can be achieved successfully if the envelope of the patterns is utilized for pattern matching.

To obtain the envelope of the patterns the differences of data samples every second from 16 to $32 \mathrm{~s}$ are used as indicated in the following formula,

$$
V_{i n}=5 \times\left(V_{n+1}-V_{n}\right) \quad(n=16,17, \ldots, 31)
$$

where $V_{\text {in }}$ means the input data for a neural network. Figure 3 shows the typical forms of the envelopes for the gas samples, which are utilized as input patterns of a neural network involved in the discrimination experiment.

A three layered BP neural network having 16 nodes in the input layer, 12 nodes in the hidden layer and 5 nodes in the output layer was used for gas discrimination. Only two patterns were selected arbitrarily as training patterns from 30 patterns of each gas sample, in other words, 10 patterns were selected out of 150 patterns for total gas samples introduced, and the training was performed until the total square error between the teaching pattern and the output patterns at the output layer was reduced to 0.05 .

Table 1 shows the results of discrimination for each introduced gas sample. Perfect discrimination was achieved for the butane, LP gas and methane, while hydrogen and town gas showed $96.7 \%$ and $93.3 \%$ discrimination rate, respectively, and the average discrimination rate for all gas samples was $98 \%$.

Comparing the results with those of previous papers ${ }^{4), 5)}$, which had revealed that the discrimination rates for
Table 1 Discrimination results for the introduced gases: B, butane; H, hydrogen; L, LP gas; M, methane; T, town gas.

\begin{tabular}{c|c|c|c|c|c|c}
\hline \hline \multicolumn{2}{c|}{} & \multicolumn{5}{|c}{ Introduced Gas } \\
\cline { 3 - 8 } \multicolumn{2}{c|}{} & B & H & L & M & T \\
\hline \multirow{4}{*}{ Discriminated } & B & 30 & 0 & 0 & 0 & 0 \\
\cline { 2 - 7 } & H & 0 & 29 & 0 & 0 & 2 \\
\cline { 2 - 7 } & $\mathrm{L}$ & 0 & 0 & 30 & 0 & 0 \\
\cline { 2 - 7 } & $\mathrm{M}$ & 0 & 0 & 0 & 30 & 0 \\
\cline { 2 - 7 } & $\mathrm{T}$ & 0 & 1 & 0 & 0 & 28 \\
\hline \multicolumn{2}{c|}{ Rate (\%) } & 100 & 96.7 & 100 & 100 & 93.3 \\
\hline \hline
\end{tabular}

the gases of similar characteristics were around $70 \%$, the discrimination capability, that is, the gas selectivity of the sensor is remarkably improved by the proposed method.

\section{Conclusion}

The periodic operation of a semiconductor gas sensor was proposed to improve the temperature-humidity dependence and gas selectivity of the sensor.

The proposed method was examined in discriminating five kinds of inflammable gases and $98 \%$ discrimination rate was achieved.

We expect that the proposed method could be applied to the concentration-independent gas sensing system as well as to the gas discrimination in a mixed atmosphere.

\section{References}

1) M. Kaneyasu, A. Ikegami, H. Arima and S. Iwanaga: Smell Identification Using a Thick-Film Hybrid Gas Sensor, IEEE Trans. on Components Hybrids and Manufacturing Technology, CHMT-10, 267/273 (1987)

2) H. Nanto, T. Kawai and S. Tsubakino: Gas Discrimination Using ZnO Thin Film Gas Sensor in Conjunction with Neural Network Pattern Recognition, Proc. of the 7th Int. Conf. on Solid-State Sensors and Actuators, Yokohama, Japan (1993)

3) M. Kanamori, K. Suzuki, Y. Ohya and Y. Takahashi: Analysis of the Change in the Carrier Concentration of $\mathrm{SnO}_{2}$ Thin Film Gas Sensor, JJAP 33, 6680/6683 (1994)

4) O. Taketoshi, K. Kobayashi, R. Konishi and H. Sasakura: Discrimination of Gas by Using Fuzzy Theory, Trans. SICE, Vol. 27, No. 5, 503/508 (1991)

5) T. Aoki, R. Konishi, H. Kimura and D. Suetsugu: The Proposal of Gas Discrimination by Using Fuzzy Reasoning, Trans. SICE, Vol. 28, No. 12, 1478/1483 (1992)

6) Y. Hiranaka, T. Abe and H. Murata: Gas-dependent response in the temperature transient of $\mathrm{SnO}_{2}$ gas sensors, Sensors and Actuators B, 9 (1992) 177/182

7) T. Seiyama, J. Shiokawa, S. Suzuki and K. Fueki: Chemical sensors, Kodansha (1991) 